\title{
Variance properties of a two-step ARX estimation procedure
}

\author{
Fredrik Tjärnström, Lennart Ljung \\ Division of Automatic Control \\ Department of Electrical Engineering \\ Linköpings universitet, SE-581 83 Linköping, Sweden \\ WWW: http://WwW. control.isy.liu.se \\ Email: fredrikt@isy.liu.se, ljung@isy.liu.se
}

24th September 2001

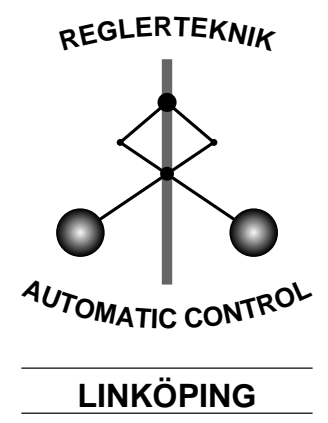

Report No.: LiTH-ISY-R-2393

Submitted to European Control Conference, September 2001,

Porto, Portugal

Technical reports from the Automatic Control group in Linköping are available by anonymous ftp at the address ftp.control.isy.liu.se. This report is contained in the file 2393.pdf. 


\begin{abstract}
In this contribution we discuss some variance properties of a two-step ARX estimation scheme. An expression for the covariance of the final low order model is calculated and it is discussed how one should minimize this covariance. The implication of the results is that identification of the dynamics of a system could very easily be performed with standard linear least squares (two times), even if the measurement noise is heavily colored. We also show a numerical example, where this two-step estimation scheme gives a variance which is close (but not equal) to the the Cramèr-Rao lower bound. Moreover, we show that the point estimate of the covariance is close to the one obtained through Monte Carlo simulations.
\end{abstract}

\title{
Keywords: Identification Methods, Estimation
}




\title{
VARIANCE PROPERTIES OF A TWO-STEP ARX ESTIMATION PROCEDURE
}

\author{
Fredrik Tjärnström and Lennart Ljung \\ Division of Automatic Control, Linkpings universitet, \\ SE-581 83 Linkping, Sweden. \\ Fax: +4613282622 \\ E-mail: \{fredrikt,ljung\}@isy.liu.se \\ Internet: http://www.control.isy.liu.se
}

Keywords: Identification Methods, Estimation

\begin{abstract}
In this contribution we discuss some variance properties of a two-step ARX estimation scheme. An expression for the covariance of the final low order model is calculated and it is discussed how one should minimize this covariance. The implication of the results is that identification of the dynamics of a system could very easily be performed with standard linear least squares (two times), even if the measurement noise is heavily colored. We also show a numerical example, where this two-step estimation scheme gives a variance which is close (but not equal) to the the Cramèr-Rao lower bound. Moreover, we show that the point estimate of the covariance is close to the one obtained through Monte Carlo simulations.
\end{abstract}

\section{Introduction}

An appealing approach to identification of a low order model is to start by estimating a high order model and then reduce it according to some specific reduction criteria. One drawback with this approach is that there has been no general theory on how to analyze the variance properties of the low order model. One of the more recent contributions are [8] (see also [7]). Here a quite general expression for the variance of the low order model is given. This expression holds if the low order model is obtained by solving an optimization problem, expressed in the high and low order models. Other approaches that take the variance of the high order model into account when performing the reduction step are $[10,9,6,11]$. The problem with these approaches is that they are restricted to a quite small class of reduction techniques and model classes.

In this paper we will analyze a two-step procedure for estimating a low order model. The idea stems from $[1,4]$. In the original idea, a high order ARX model is estimated from data. A noise free simulation (using the same input signal) is then performed. From the simulated data a low order ARX-model is estimated. The change we have introduced is to use an arbitrary input signal in the simulation step, both in terms of its color and of its length. These changes has led us to rewrite the problem as a minimization problem, where the input color in the second step is a design variable.
The organization of the paper is as follows. Section 2 presents the identification setup, with the studied model class. In Section 3 we present some tools to analyze the covariance of the low order model. Guidelines for the user in the model reduction step are presented in Section 5 and the implementation issues are discussed in Section 6. In Section 7 the results are verified by simulations. Section 8 concludes the paper.

\section{Identification setup}

Here we give a brief summary of some identification results that we will make use of. See [3] for further details.

In this contribution we will estimate systems that can be described by the ARX structure

$$
A(q, \theta) y(t)=B(q, \theta) u(t)+e(t),
$$

where

$$
\begin{aligned}
A(q, \theta) & =1+a_{1} q^{-1}+\ldots+a_{n_{a}} q^{-n_{a}}, \\
B(q, \theta) & =b_{1} q^{-n_{k}}+\ldots+b_{n_{b}} q^{-n_{k}-n_{b}+1} \\
\theta & =\left(\begin{array}{llllll}
a_{1} & \ldots & a_{n_{a}} & b_{1} & \ldots & b_{n_{b}}
\end{array}\right)^{T} .
\end{aligned}
$$

Here $e(t)$ is a white noise process with variance $\lambda$ and $u(t)$ is the input signal with spectrum,

$$
\Phi_{u}(\omega)=\left|Q\left(e^{i \omega}\right)\right|^{2} .
$$

A total of $N$ input-output data are collected.

Rewriting (1) yields

$$
y(t)=\varphi(t)^{T} \theta+e(t),
$$

where

$$
\begin{aligned}
\varphi(t)= & \left(-y(t-1), \ldots,-y\left(t-n_{a}\right),\right. \\
& \left.u\left(t-n_{k}\right), \ldots, u\left(t-n_{k}-n_{b}+1\right)\right)^{T} .
\end{aligned}
$$

The parameters $\theta$ are estimated by minimizing the squared sum of the prediction errors

$$
\begin{aligned}
& V_{N}(\theta)=\frac{1}{N} \sum_{t=1}^{N} \varepsilon^{2}(t, \theta) \\
& \varepsilon(t, \theta)=y(t)-\hat{y}(t \mid \theta)=y(t)-\varphi^{T}(t) \theta .
\end{aligned}
$$


The estimate $\hat{\theta}_{N}$ is then given by

$$
\begin{aligned}
\hat{\theta}_{N} & =\arg \min V_{N}(\theta) \\
& =\left[\frac{1}{N} \sum_{t=1}^{N} \varphi(t) \varphi^{T}(t)\right]^{-1} \frac{1}{N} \sum_{t=1}^{N} \varphi(t) y(t)
\end{aligned}
$$

Assuming that the true system can be described with an ARX model, i.e., that data is generated according to

$$
A(q) y(t)=B(q) u(t)+e(t)
$$

where for some $\theta_{0}$

$$
\begin{aligned}
& A(q)=A\left(q, \theta_{0}\right), \\
& B(q)=B\left(q, \theta_{0}\right),
\end{aligned}
$$

we have $[3$, Chapter 8$]$ that

$$
\hat{\theta}_{N} \rightarrow \theta_{0} \text { as } N \rightarrow \infty .
$$

The covariance of $\hat{\theta}_{N}$ is given by

$$
\operatorname{Cov} \hat{\theta}_{N}=\lambda_{0}\left[\overline{\mathrm{E}} \varphi(t) \varphi^{T}(t)\right]^{-1},
$$

where $\overline{\mathrm{E}} f(t)=\lim _{N \rightarrow \infty} \frac{1}{N} \sum_{t=1}^{N} \operatorname{E} f(t)$.

We also mention that if the model order, $n=n_{a}=n_{b}$, tends to infinity we have the following result

$$
\operatorname{Cov} \frac{B\left(e^{i \omega}, \hat{\theta}_{N}\right)}{A\left(e^{i \omega}, \hat{\theta}_{N}\right)} \approx \frac{n}{N} \frac{\Phi_{v}(\omega)}{\Phi_{u}(\omega)} \text { as } N, n, \frac{N}{n} \rightarrow \infty,
$$

where $\Phi_{v}(\omega)$ is the noise spectrum. In this case $\Phi_{v}(\omega)=$ $\lambda /\left|A\left(e^{i \omega}\right)\right|^{2}$. That is the variance of the frequency function is (asymptotically) proportional to the noise-to-signal ratio.

One final remark on ARX models is that they can approximate any linear system arbitrarily well, just use a high enough model order. This can be useful as a modeling tool itself; estimate a high-order ARX model and reduce it to an appropriate structure using some model reduction technique.

\section{Model Reduction}

This section contains a brief summary of some model reduction results presented in $[8,7]$.

Assume that we have high order model $\hat{\theta}$ with covariance $\operatorname{Cov} \hat{\theta}$. This model is subjected to model reduction described by

$$
\hat{\eta}=\arg \min _{\eta} V(\eta, \hat{\theta})
$$

where we assume that $V$ is two times continuously differentiable. Then the low order model $\hat{\eta}$ has an asymptotic covariance given by

$$
\begin{aligned}
\operatorname{Cov} \hat{\eta} \approx & {\left[V_{\eta \eta}^{\prime \prime}\left(\eta^{*}, \theta^{*}\right)\right]^{-1}\left[V_{\eta \theta}^{\prime \prime}\left(\eta^{*}, \theta^{*}\right)\right] } \\
& \times \operatorname{Cov} \hat{\theta} \\
& \times\left[V_{\theta \eta}^{\prime \prime}\left(\eta^{*}, \theta^{*}\right)\right]\left[V_{\eta \eta}^{\prime \prime}\left(\eta^{*}, \theta^{*}\right)\right]^{-1} .
\end{aligned}
$$

Here $\eta^{*}$ and $\theta^{*}$ are the limiting estimates (as the number of data tends to infinity). If the model class contains the true system then the limiting values equal the true parameters, i.e., $\eta^{*}=$ $\eta_{0}, \theta^{*}=\theta_{0}$.

In the $[8,7]$ this expression was used to analyze the variance properties of $L_{2}$ model reduction. The basic results were:

- when estimating a finite impulse response models of low order, it could in some cases be strictly better to estimate the low order model by model reduction of a high order model compared with estimating the low order model directly.

- when estimating an output error model we will not lose anything in terms of variance when getting the estimate from a reduced high order model compared to estimating the low order model directly from data.

\section{Estimation procedure}

The systems that we model in this contribution are described by the ARARX structure

$$
A(q) y(t)=B(q) u(t)+\frac{1}{D(q)} e(t) .
$$

To estimate the parameters in this model structure one typically uses non-linear optimization methods [3]. Other approaches to this are described in $[6,1]$. Here we will follow the some ideas that are used in $[1,11]$. We point out that the aim of the paper is analyze the variance properties of the estimation of $A$ and $B$ using this procedure. At the same time we discuss if this procedure could be optimized to achieve lower variance.

To be able to estimate (16) using linear least squares we rewrite it according to

$$
\begin{aligned}
A(q) y(t) & =B(q) u(t)+\frac{1}{D(q)} e(t), \\
& \Leftrightarrow \\
D(q) A(q) y(t) & =D(q) B(q) u(t)+e(t), \\
& \Leftrightarrow \\
\tilde{A}(q) y(t) & =\tilde{B}(q) u(t)+e(t) .
\end{aligned}
$$

That is, we get an ARX system in $\tilde{A}, \tilde{B}$. The first step in the two-step procedure is to compute the ARX estimate, $\hat{\theta}$, of (19) with

$$
\theta=\left(\begin{array}{lllll}
\tilde{a}_{1} & \ldots \tilde{a}_{\tilde{n}_{a}} & \tilde{b}_{1} & \ldots \tilde{b}_{\tilde{n}_{b}}
\end{array}\right)
$$

This gives us the initial unbiased high order estimates $\tilde{A}(q, \hat{\theta})$ and $\tilde{B}(q, \hat{\theta})$. The covariance of $\hat{\theta}$ is given by (12), i.e.,

$$
\operatorname{Cov} \hat{\theta} \approx \frac{\lambda}{N}\left[\overline{\mathrm{E}} \varphi(t) \varphi^{T}(t)\right]^{-1}
$$

Denote the high order model by

$$
\hat{\tilde{A}}(q)=\tilde{A}(q, \hat{\theta}), \quad \hat{\tilde{B}}(q)=\tilde{B}(q, \hat{\theta}) .
$$


Now parameterize the low order model $(A(q)$ and $B(q))$ with

$$
\eta=\left(\begin{array}{llllll}
a_{1} & \ldots & a_{n_{a}} & b_{1} & \ldots & b_{n_{b}}
\end{array}\right)^{T}
$$

Estimate $\eta$ by minimizing

$$
V(\eta, \hat{\theta})=\overline{\mathrm{E}}\left(\varphi_{h}^{T}(t, \hat{\theta}) \hat{\theta}-\varphi_{l}^{T}(t, \hat{\theta}) \eta\right)^{2}
$$

Here $\varphi_{h}(t, \hat{\theta})$ and $\varphi_{l}(t, \hat{\theta})$ are regression vectors of appropriate size, built from $u_{s}(t)$ and $y_{s}(t)$, where $u_{s}(t)$ has the spectrum

$$
\Phi_{u_{s}}(\omega)=\left|L\left(e^{i \omega}\right)\right|^{2} \Phi_{u}(\omega),
$$

and $y_{s}(t)$ is generated according to

$$
y_{s}(t)=\frac{\hat{\tilde{B}}(q)}{\hat{\tilde{A}}(q)} u_{s}(t) .
$$

The loss function (24) is chosen to make the reduction step easy. Since (24) is a quadratic criterion, the estimate $\hat{\eta}$ becomes

$$
\hat{\eta}=\left(\overline{\mathrm{E}} \varphi_{l}(t, \hat{\theta}) \varphi_{l}^{T}(t, \hat{\theta})\right)^{-1}\left(\overline{\mathrm{E}} \varphi_{l}(t, \hat{\theta}) \varphi_{h}^{T}(t, \hat{\theta})\right) \hat{\theta}
$$

Asymptotically (in $N$ ) it then holds (since $\hat{\theta}$ is asymptotically unbiased) that

$$
\begin{aligned}
\eta_{0} & =\lim _{N \rightarrow \infty} \hat{\eta} \\
& =\left(\overline{\mathrm{E}} \varphi_{l}(t, \theta) \varphi_{l}^{T}(t, \theta)\right)^{-1}\left(\overline{\mathrm{E}} \varphi_{l}(t, \theta) \varphi_{h}^{T}(t, \theta)\right) \theta_{0} .
\end{aligned}
$$

The procedure above gives us the low order estimates $\hat{A}(q)=$ $A(q, \hat{\eta})$ and $\hat{B}(q)=B(q, \hat{\eta})$. Note here that since $\hat{\tilde{B}} / \hat{\tilde{A}}$ is a consistent estimate of $\tilde{B} / \tilde{A}$, it holds that $\hat{B} / \hat{A}$ is a consistent estimate of $B / A$, irrespectively of the choice of input-prefilter $L(q)$.

The main difficulty lies in the calculation of the asymptotic covariance of the low order model, $\eta$. It is important to note that the covariance of $\hat{\eta}$ does not equal

$$
\left(\overline{\mathrm{E}} \varphi_{l} \varphi_{l}^{T}\right)^{-1}\left(\overline{\mathrm{E}} \varphi_{l} \varphi_{h}^{T}\right) \operatorname{Cov} \hat{\theta}\left(\overline{\mathrm{E}} \varphi_{h} \varphi_{l}^{T}\right)\left(\overline{\mathrm{E}} \varphi_{l} \varphi_{l}^{T}\right)^{-1}
$$

due to the dependency on $\hat{\theta}$ in $\varphi_{l}$ and $\varphi_{h}$.

To get a correct (asymptotic) expression we have to compute $V_{\eta \eta}^{\prime \prime}$ and $V_{\eta \theta}^{\prime \prime}$ in the limiting point $\eta_{0}, \theta_{0}$, as described in Section 3. From (24) we get (using shorthand notation $\varphi_{l}(\theta)=$ $\left.\varphi_{l}(t, \theta)\right)$

$$
\begin{aligned}
V(\eta, \theta) & =\overline{\mathrm{E}}\left(\varphi_{l}^{T}(\theta) \eta-\varphi_{h}^{T}(\theta) \theta\right) \\
V_{\eta}^{\prime}(\eta, \theta) & =\overline{\mathrm{E}} \varphi_{l}(\theta)\left(\varphi_{l}^{T}(\theta) \eta-\varphi_{h}^{T}(\theta) \theta\right) \\
V_{\eta \eta}^{\prime \prime}\left(\eta_{0}, \theta_{0}\right) & =\overline{\mathrm{E}} \varphi_{l}\left(\theta_{0}\right) \varphi_{l}^{T}\left(\theta_{0}\right) \\
V_{\theta \eta}^{\prime \prime}(\eta, \theta) & =\overline{\mathrm{E}} \varphi_{l}^{\prime}(\theta)\left(\varphi_{l}^{T}(\theta) \eta-\varphi_{h}^{T}(\theta) \theta\right) \\
& +\overline{\mathrm{E}} \varphi_{l}(\theta)\left(\eta^{T} \varphi_{l}^{\prime}(\theta)-\theta^{T} \varphi_{h}^{\prime}(\theta)-\varphi_{h}^{T}(\theta)\right) \\
V_{\theta \eta}^{\prime \prime}\left(\eta_{0}, \theta_{0}\right) & =\overline{\mathrm{E}} \varphi_{l}\left(\theta_{0}\right)\left(\eta_{0}^{T} \varphi_{l}^{\prime}\left(\theta_{0}\right)-\theta_{0}^{T} \varphi_{h}^{\prime}\left(\theta_{0}\right)-\varphi_{h}^{T}\left(\theta_{0}\right)\right),
\end{aligned}
$$

where the last equality follows from the fact that $\varphi_{l}^{T}\left(\theta_{0}\right) \eta_{0}=$ $\varphi_{h}^{T}\left(\theta_{0}\right) \theta_{0}$.

Now (30) has to be calculated. In the following we will write $\hat{\tilde{A}}$ for $\hat{\tilde{A}}(q)$ to ease the notation. First we have

$$
\begin{aligned}
& \varphi_{h}^{T}(t, \hat{\theta})=\left(-\frac{\hat{\tilde{B}}}{\hat{\tilde{A}}} u_{s}(t-1) \ldots-\frac{\hat{\tilde{B}}}{\hat{\tilde{A}}} u_{s}\left(t-\tilde{n}_{a}\right)\right. \\
& \left.u_{s}\left(t-n_{k}\right) \ldots u_{s}\left(t-n_{k}-\tilde{n}_{b}+1\right)\right)
\end{aligned}
$$

The derivative of (31) with respect to $\theta$ evaluated at the optimum, $\theta_{0}$, becomes (see top of next page, Eqn. (32)). A similar expression holds for the derivative of $\varphi_{l}(t, \theta)$ with respect to $\theta$.

Moreover

$$
\begin{gathered}
\theta_{0}^{T} \varphi_{h}^{\prime}\left(t, \theta_{0}\right)=\left(\frac{\tilde{B}(\tilde{A}-1)}{\tilde{A}^{2}} u_{s}(t-1) \ldots \frac{\tilde{B}(\tilde{A}-1)}{\tilde{A}} u_{s}\left(t-\tilde{n}_{a}\right)\right. \\
\left.-\frac{\tilde{A}-1}{\tilde{A}} u_{s}\left(t-n_{k}\right) \ldots-\frac{\tilde{A}-1}{\tilde{A}} u_{s}\left(t-n_{k}-\tilde{n}_{b}+1\right)\right) \\
=-\frac{\tilde{A}-1}{\tilde{A}} \varphi_{h}^{T}\left(t, \theta_{0}\right)
\end{gathered}
$$

and

$$
\begin{array}{r}
\eta_{0}^{T} \varphi_{l}^{\prime}\left(t, \theta_{0}\right)=\left(\frac{\tilde{B}(A-1)}{\tilde{A}^{2}} u_{s}(t-1) \ldots \frac{\tilde{B}(A-1)}{\tilde{A}} u_{s}\left(t-\tilde{n}_{a}\right)\right. \\
\left.-\frac{A-1}{\tilde{A}} u_{s}\left(t-n_{k}\right) \ldots-\frac{A-1}{\tilde{A}} u_{s}\left(t-n_{k}-\tilde{n}_{b}+1\right)\right) \\
=-\frac{A-1}{\tilde{A}} \varphi_{h}^{T}\left(t, \theta_{0}\right)
\end{array}
$$

Now using (33) and (34) we get

$$
\begin{aligned}
& \eta_{0}^{T} \varphi_{h}^{\prime}\left(t, \theta_{0}\right)-\eta_{0}^{T} \varphi_{l}^{\prime}\left(t, \theta_{0}\right)-\varphi_{h}^{T}\left(t, \theta_{0}\right)= \\
& -\frac{A-1}{\tilde{A}} \varphi_{h}^{T}\left(t, \theta_{0}\right)+\frac{\tilde{A}-1}{\tilde{A}} \varphi_{h}^{T}\left(t, \theta_{0}\right)-\varphi_{h}^{T}\left(t, \theta_{0}\right) \\
& \quad=-\frac{A}{\tilde{A}} \varphi_{h}^{T}\left(t, \theta_{0}\right)=-\frac{1}{D} \varphi_{h}^{T}\left(t, \theta_{0}\right)
\end{aligned}
$$

where the last equality follows since $\tilde{A}=A D$ in the limit.

Putting (15), (29), (30), and (35) together we get the following (rather complicated) expression for the covariance of the low order model

$$
\begin{aligned}
\operatorname{Cov} \hat{\eta} \approx & \left(\overline{\mathrm{E}} \varphi_{l}\left(t, \theta_{0}\right) \varphi_{l}^{T}\left(t, \theta_{0}\right)\right)^{-1} \\
& \times\left(\overline{\mathrm{E}} \varphi_{l}\left(t, \theta_{0}\right) \frac{1}{D(q)} \varphi_{h}^{T}\left(t, \theta_{0}\right)\right) \\
& \times \frac{\lambda}{N}\left(\overline{\mathrm{E}} \varphi(t) \varphi^{T}(t)\right)^{-1} \\
& \times\left(\overline{\mathrm{E}} \frac{1}{D(q)} \varphi_{h}\left(t, \theta_{0}\right) \varphi_{l}^{T}\left(t, \theta_{0}\right)\right) \\
& \times\left(\overline{\mathrm{E}} \varphi_{l}\left(t, \theta_{0}\right) \varphi_{l}^{T}\left(t, \theta_{0}\right)\right)^{-1} .
\end{aligned}
$$




$$
\varphi_{h}^{\prime}\left(t, \theta_{0}\right)=\left(\begin{array}{ccc}
\frac{\hat{\tilde{B}}}{\hat{\tilde{A}}^{2}} u_{s}(t-2) & \ldots & \frac{\hat{\tilde{B}}}{\hat{\tilde{A}}^{2}} u_{s}\left(t-\tilde{n}_{a}-1\right) \\
\vdots & & \vdots \\
\frac{\hat{\tilde{B}}}{\hat{\tilde{A}}^{2}} u_{s}\left(t-\tilde{n}_{a}-1\right) & \ldots & \frac{\hat{\tilde{B}}}{\hat{\tilde{A}}^{2}} u_{s}\left(t-2 \tilde{n}_{a}\right) \\
0 & \ldots & 0 \\
\vdots & & \\
0 & \ldots & 0
\end{array}\right.
$$$$
\left.\begin{array}{ccc}
-\frac{1}{\tilde{\hat{A}}} u_{s}\left(t-n_{k}-1\right) & \ldots & -\frac{1}{\tilde{\hat{A}}} u_{s}\left(t-n_{k}-\tilde{n}_{b}\right) \\
\vdots & & \vdots \\
-\frac{1}{\tilde{\hat{A}}} u_{s}\left(t-n_{k}-\tilde{n}_{a}\right) & \ldots & -\frac{1}{\tilde{\hat{A}}} u_{s}\left(t-n_{k}-\tilde{n}_{b}-\tilde{n}_{a}+1\right) \\
0 & \ldots & 0 \\
0 & \ldots & \vdots
\end{array}\right)
$$

\section{Choice of prefilter}

Looking at expression (36) one interesting question arises: How should the input prefilter $L(q)$ be chosen to minimize the variance of the low order estimate? This question has no obvious solution, but one might think of one of the following candidates

$$
L(q)=H(q) \approx \frac{1}{\hat{\tilde{A}}(q)} ; \quad L(q)=H^{-1}(q) \approx \hat{\tilde{A}}(q) .
$$

Here the first filter corresponds to the idea of using input energy in relation to the asymptotic variance of the estimate (which is proportional to noise-to-signal ratio, i.e., $\Phi_{v}(\omega) / \Phi_{u}(\omega)$. On the other hand the second filter corresponds to the inverse of this. The idea behind this is to use a lot of energy where the initial estimate is good. However, non of these ideas are correct.

Looking back, we note that the low order model predicts the "simulated" output as (using (26))

$$
\begin{aligned}
\hat{y}_{s}(t) & =\varphi_{l}^{T}(t, \hat{\theta}) \eta \\
& =(1-A(\eta)) y_{s}(t)+B(\eta) u_{s}(t) \\
& =\left((1-A(\eta)) \frac{\hat{\tilde{B}}}{\hat{\tilde{A}}}+B(\eta)\right) u_{s}(t) .
\end{aligned}
$$

Putting (24),(26), and (38) together gives

$$
V(\eta, \hat{\theta})=\overline{\mathrm{E}}\left[\frac{B(\eta) \hat{\tilde{A}}-A(\eta) \hat{\tilde{B}}}{\hat{\tilde{A}}} u_{s}(t)\right]^{2} .
$$

Introducing the notation $G(\eta)=B(\eta) / A(\eta)$ and $\hat{\tilde{G}}=\hat{\tilde{B}} / \hat{\tilde{A}}$, this can be rewritten as

$$
V(\eta, \hat{\theta})=\overline{\mathrm{E}}\left[(G(\eta)-\hat{\tilde{G}}) A(\eta) u_{s}(t)\right]^{2} .
$$

Finally using Parsevals formula and (25) gives

$$
\begin{aligned}
V(\eta, \hat{\theta})=\frac{1}{2 \pi} \int_{0}^{2 \pi} & \left|G\left(e^{i \omega}, \eta\right)-\hat{\tilde{G}}\left(e^{i \omega}\right)\right|^{2} \\
& \times\left|A\left(e^{i \omega}, \eta\right) L\left(e^{i \omega}\right)\right|^{2} \Phi_{u}(\omega) d \omega
\end{aligned}
$$

This form of the the loss function gives more insight in how the low order estimate is obtained.
Now studying (41) it is clear that last factor $\left|A\left(e^{i \omega}, \eta\right) L\left(e^{i \omega}\right)\right|^{2} \Phi_{u}(\omega)$ equals $\Phi_{u}(\omega) / \Phi_{v}(\omega)$ if

$$
L\left(e^{i \omega}\right)=D\left(e^{i \omega}\right)
$$

This would correspond to minimizing the distance between $G\left(e^{i \omega}, \eta\right)$ and $\hat{\tilde{G}}\left(e^{i \omega}\right)$ weighted in the inverse of the variance of $\hat{\tilde{G}}\left(e^{i \omega}\right)$ according to (13). One should note that this result is based on (13), which is asymptotic in both data and model order. However, it gives some insight in how the prefilter should be chosen. This is very similar to the idea that is used in [11, Section 7.2].

\section{Implementation}

- computing the expectations that are needed to obtain the estimate of $\eta$ in (27) and the covariance of $\hat{\eta}$ in (36).

- estimating the filter $D(q)$ that is needed in to compute the covariance of $\hat{\eta}$ in (36).

- estimating the filter $D(q)$ that might be used as input prefilter in (42).

A simple solution to the first problem is to approximate the expectation with the finite sample mean, i.e.,

$$
\overline{\mathrm{E}} f(t)=\lim _{N \rightarrow \infty} \frac{1}{N} \sum_{t=1}^{N} \mathrm{E} f(t) \approx \frac{1}{N} \sum_{t=1}^{N} f(t) .
$$

This is done by simulating the output according to (26), with an input sequence, $u_{s}(t)$, of length $\tilde{N}$, where $\tilde{N} \gg N$. From the simulated inputs and outputs the regressors $\varphi_{l}(t)$ and $\varphi_{h}(t)$ are constructed.

For the second problem it is natural to use the approximation

$$
\frac{1}{D(q)} \approx \frac{\hat{A}(q)}{\hat{\tilde{A}}(q)}
$$

according to the last equality in (35).

The third problem is more difficult, since (in contrast to the second problem) $\hat{A}$ is not available when the prefilter is to be
The implementation problems are: 
computed. Then one has to find the common zeros of $\hat{\tilde{A}}(q)$ and $\hat{\tilde{B}}(q)$. This can be by the technique suggested in [5]. Then the zeros of $D(q)$ are estimated as a (weighted) mean of the zeros in $\hat{\tilde{A}}(q)$ and $\hat{\tilde{B}}(q)$.

\section{Numerical example}

Let the data be generated according to

$$
\begin{aligned}
A(q) y(t) & =B(q) u(t)+\frac{1}{D(q)} e(t), \\
A(q) & =\left(1-0.2 q^{1}\right)\left(1-0.3 q^{-1}\right) \\
B(q) & =q^{-1}\left(1-0.7 q^{-1}\right) \\
D(q) & =1+0.95 q^{-1} .
\end{aligned}
$$

i.e.,

$$
\begin{aligned}
\tilde{A}(q) y(t) & =\tilde{B}(q) u(t)+e(t) \\
\tilde{A}(q) & =\left(1-0.2 q^{1}\right)\left(1-0.3 q^{-1}\right)\left(1+0.95 q^{-1}\right) \\
\tilde{B}(q) & =q^{-1}\left(1-0.7 q^{-1}\right)\left(1+0.95 q^{-1}\right) .
\end{aligned}
$$

The input, $u(t)$, and the noise, $e(t)$, are Gaussian white noise sequences with variances 1 and 0.001 respectively. $N=500$ data samples are generated. Initially an ARX model with $\tilde{n}_{a}=$ $\tilde{n}_{b}=3$ is estimated.

This model is then reduced to order $n_{a}=n_{b}=2$ according to an approximate finite sample version of (24) using an input sequence consisting of 25000 samples (i.e., $\tilde{N}=50 \mathrm{~N}$ ). The input is generated as Gaussian white noise with variance 0.001 , filtered through three different prefilters $L_{1}(q)=\hat{D}(q)$, $L_{2}(q)=\hat{\tilde{A}}(q)=\hat{H}^{-1}(q)$, and $L_{3}(q)=1 / \hat{\tilde{A}}(q)=\hat{H}(q)$. The ARARX model (estimation of the $A, B$ and $D$ polynomial) is also computed using System Identification Toolbox [2] (the iterative searches giving these estimates were initialized in their asymptotic optima). Note that the ARARX estimation meets the Cramér-Rao bound asymptotically. This procedure was then repeated 1000 times and the mean and the empirical covariance estimates was computed. The means of estimated systems are presented in Table 1 and covariance estimates are presented in Table 2 .

\begin{tabular}{|c|cccc|}
\hline & $a_{1}$ & $a_{2}$ & $b_{1}$ & $b_{2}$ \\
\hline \hline True values & -0.5 & 0.06 & 1 & 0.7 \\
\hline ARARX & -0.5000 & 0.0599 & 1.0001 & -0.7001 \\
\hline$L_{1}=\hat{D}$ & -0.5000 & 0.0599 & 1.0001 & -0.7001 \\
\hline$L_{2}=1 / \hat{H}=\hat{\tilde{A}}$ & -0.5000 & 0.0599 & 1.0001 & -0.7001 \\
\hline$L_{3}=\hat{H}=1 / \hat{\tilde{A}}$ & -0.4384 & 0.0646 & 0.9985 & -0.6401 \\
\hline
\end{tabular}

Table 1: Obtained sample mean estimates over 1000 realizations for three different types of prefilters together with the estimates for an ARARX model. The top row contains the true values.

It is clear from Table 1 and 2 that it is possible to obtain

\begin{tabular}{|c|cccc|}
\hline & $a_{1}$ & $a_{2}$ & $b_{1}$ & $b_{2}$ \\
\hline \hline \multirow{3}{*}{ ARARX } & 6.72 & 1.55 & -0.13 & 6.89 \\
& 1.55 & 1.24 & -0.30 & 2.17 \\
& -0.13 & -0.30 & 0.86 & -0.70 \\
& 6.89 & 2.17 & -0.70 & 8.18 \\
\hline \multirow{3}{*}{$L_{1}=\hat{D}$} & 6.85 & 1.58 & -0.14 & 7.02 \\
& 1.58 & 1.25 & -0.31 & 2.20 \\
& -0.14 & -0.31 & 0.86 & -0.71 \\
$L_{2}=1 / \hat{H}=\hat{\tilde{A}}$ & 7.02 & 2.20 & -0.71 & 8.31 \\
\hline \multirow{3}{*}{$L_{3}=\hat{H}=1 / \hat{\tilde{A}}$} & 7.19 & 1.71 & -0.05 & 7.38 \\
& 1.71 & 1.31 & -0.27 & 2.34 \\
& -0.05 & -0.27 & 0.88 & -0.61 \\
& 7.38 & 2.34 & -0.61 & 8.69 \\
\hline & 6661 & 794 & -173 & 6598 \\
& -173 & 246 & -27.2 & 847 \\
& 6598 & 847 & -174 & 6561 \\
\hline
\end{tabular}

Table 2: Obtained sample covariance estimates over 1000 realizations for three different types of prefilters together with the covariance estimates for the ARARX model. All covariance matrices are normalized by the factor $N / \lambda$.

very accurate estimates of the low order model using the proposed two-step procedure. We see that first two prefilters all give results that are comparable to the ARARX estimation. Moreover, we see that "bad" choices of prefilters exists. Here $L_{3}=\hat{H}=1 / \hat{A}$ gives very uncertain estimates.

It is an interesting fact that the final covariance of the low order model seems to be quite robust to variations in the prefilter. The first two filters have rather different interpretations, but both give approximately the same covariance in the final estimate. The thing that connects them is that they both happen to have a low-pass character with similar bandwidths. This "robustification" towards variations in $\hat{D}$ is very positive due to the difficulties in estimating it.

It is also interesting to see how good covariance estimates it is possible to obtain using (36). In Table 3 we present point estimates of the parameters and the covariances using (36). In all three cases the prefilter $L_{1}(q)=\hat{D}(q)$ was used. The experiments where performed using $N=500,1000,5000$ (and $\tilde{N}=50 N$ ) data respectively. From the table we see that the estimates are in good agreement with what is obtained using Monte Carlo simulations in Table 2.

\section{Conclusions}

We have presented a two-step ARX estimation scheme to obtained accurate estimates of the system dynamics. In connection to this we have given an expression for the asymptotic variance of the low order model (36). In case of high model orders it was clear how to chose of prefilter to obtain optimal covariance. We have also shown that it is possible to obtain 


\begin{tabular}{|c|cccc|}
\hline & $a_{1}$ & $a_{2}$ & $b_{1}$ & $b_{2}$ \\
\hline \hline$N=500$ & -0.5017 & 0.0609 & 1.0008 & -0.7020 \\
\hline \hline & 6.56 & 1.38 & 0.06 & 6.51 \\
& 1.38 & 1.20 & -0.25 & 1.97 \\
& 0.06 & -0.25 & 0.79 & -0.49 \\
& 6.51 & 1.97 & -0.49 & 7.58 \\
\hline \hline$N=1000$ & -0.5004 & 0.0596 & 1.0006 & -0.7002 \\
\hline \hline & 6.23 & 1.35 & -0.01 & 6.26 \\
& 1.35 & 1.17 & -0.30 & 1.97 \\
& -0.01 & -0.30 & 0.77 & -0.57 \\
& 6.26 & 1.97 & -0.57 & 7.39 \\
\hline \hline$N=5000$ & -0.5011 & 0.0594 & 1.0006 & -0.7015 \\
\hline & 6.92 & 1.46 & 0.00 & 6.99 \\
& 1.46 & 1.21 & -0.31 & 2.09 \\
& 0.00 & -0.31 & 0.81 & -0.56 \\
& 6.99 & 2.09 & -0.56 & 8.17 \\
\hline
\end{tabular}

Table 3: Point estimates of the parameters, $\eta^{T}=\left(\begin{array}{llll}a_{1} & a_{2} & b_{1} & b_{2}\end{array}\right)$, of the low order model and the point estimate of their covariance matrices using (36). The estimates are obtained using $N=500,1000$, and 5000 data respectively. Line 1, 6, and 11 contains the estimated parameters and lines $2-5,7-10$, and $12-15$ contains their covariance matrices. All covariance matrices are normalized by the factor $N / \lambda$.

very good variance properties using this two-step procedure in a simple simulation example. A still open question is if there exists a prefilter $L(q)$ such that the covariance of the low order model asymptotically meets the Cramèr-Rao bound.

Final comments: Looking back at the derivations shown in this paper, we see that the results can be generalized to a wider class of systems. It turns out that we can handle systems of the form

$$
y(t)=\frac{B(q)}{F(q)} u(t)+\frac{C(q)}{D(q)} e(t),
$$

where $B, C, D$, and $F$ are all finite impulse response filters, and the last three are monic. The idea is to estimate (46) with a high order ARX model, which will then be unbiased, see Section 2. Going through all calculations we see that the only modifications that need to be done are replacing $D(q)$ in (36) and (42) with $\tilde{A}(q) / F(q)$. This means that we can still estimate the covariance of the low order model and moreover we get an expression for the optimal input prefilter.

\section{References}

[1] T. C. Hsia. Identification: Least Squares Methods. Lexington Books, Lexington, Mass., 1977.

[2] L. Ljung. The System Identification Toolbox: The Manual. The Mathworks Inc., 24 Prime Park Way, Natick, MA, 4th edition, 1995.
[3] L. Ljung. System Identification: Theory for the User. Prentice-Hall, Upper Saddle River, NJ, 2nd edition, 1999.

[4] R. N. Pandya. A class of bootstrap estimators and their relationship to generalized two stage least squares estimators. IEEE Transactions on Automatic Control, 19(6):831-835, 1974.

[5] T. Söderström. Test of pole-zero cancellation in estimated models. Automatica, 11:537-539, 1975.

[6] T. Söderström, P. Stoica, and B. Friedlander. An indirect prediction error method for system identification. Automatica, 27:183-188, 1991.

[7] F. Tjärnström. Quality estimation of approximate models. Technical Report Licentiate Thesis No. 810, Department of Electrical Engineering, Linköpings universitet, SE-581 83 Linköping, Sweden, Feb 2000.

[8] F. Tjärnström and L. Ljung. $L_{2}$-model reduction and variance reduction. In Proc IFAC Symposium, SYSID 2000, pages FrAM2-5, Santa Barbara, Jun 2000.

[9] B. Wahlberg. On the Identification and Approximation of Linear Systems. Phd thesis 163, Department of Electrical Engineering, Linköping University, 1987.

[10] B. Wahlberg. Model reduction of high order estimated models: The asymptotic ML approach. Int. J. Control, 49(1):169-192, 1989.

[11] Y. Zhu and T. Backx. Identification of Multivariable Industrial Processes for Simulation, Diagnosis and Control. Springer-Verlag, 1993. 